\title{
The Dawn of Galaxies: Deep MAMBO Imaging Surveys
}

\author{
F. Bertoldi, K.M. Menten, E. Kreysa \\ Max-Planck-Institut für Radioastronomie, 53121 Bonn, Germany \\ C.L. Carilli, F. Owen \\ NRAO, Socorro, NM 87801, USA
}

\begin{abstract}
We discuss results from sensitive, wide-field imaging of the millimeter extragalactic background using the Max-Planck Millimeter Bolometer array (MAMBO) at the IRAM $30 \mathrm{~m}$ telescope.
\end{abstract}

The detection of a far-infrared and sub-millimeter wavelength background by COBE, and the first sensitive, high-resolution SCUBA images of the sub-mm background have caused significant revisions of our picture of the star-formation history of the universe. SCUBA at $850 \mu \mathrm{m}$ discovered a population of what appear to be star forming galaxies at high redshifts, most of which are invisible at optical and near-IR wavelengths (Smail et al. 1997; Hughes et al. 1998; Barger et al. 1999; Eales et al. 1999; Bertoldi et al. 2000). The infrared luminosities of these objects are comparable to the bolometric luminosities of QSOs, but their optical faintness shows that, unlike for QSOs, nearly all of the bolometric luminosity arises from thermal emission of dust grains. The emitting dust is probably heated in massive, optically obscured star forming regions, with star formation rates $\sim 10^{3} M_{\odot} y r^{-1}$. The objects discovered with SCUBA and MAMBO can account for the integrated (sub)mm background, and thereby about $25 \%$ of the total infrared background radiation. The relation between metal production and the extragalactic background (Eales et al. 1999) would then imply that at least a quarter of all stars were formed during the extreme starbursts we now see as thermal background sources.

The total number of (sub)mm background sources discovered with SCUBA at $850 \mu \mathrm{m}(350 \mathrm{GHz})$, and with the 37-channel MAMBO array at $1.2 \mathrm{~mm}(250$ $\mathrm{GHz}$ ) now exceeds 100 , and great efforts have been made trying to identify the class of known objects to which these (sub)mm sources belong. However, most of them defy a clear optical identification, and thereby an accurate redshift determination. The positional accuracies of the SCUBA and MAMBO sources are of order $5^{\prime \prime}$, which for each source allows for a number of faint optical and near-IR sources as possible counterparts, objects which are usually too faint for spectroscopic studies. To date, only two MAMBO sources have clear near-IR identifications, and three SCUBA sources have clear optical or near-IR identifications with a spectroscopic redshift (Lilly et al. 1999; Ivison et al. 2000).

More promising are recent attempts to identify the (sub)mm background sources with radio sources in deep VLA images. We found that the majority of several dozen brightest and highest-S/N MAMBO sources in our $100 \mathrm{arcmin}^{2}$ map of the Abell 2125 region have $20 \mathrm{~cm}$ radio counterparts within $5^{\prime \prime}$ of the 


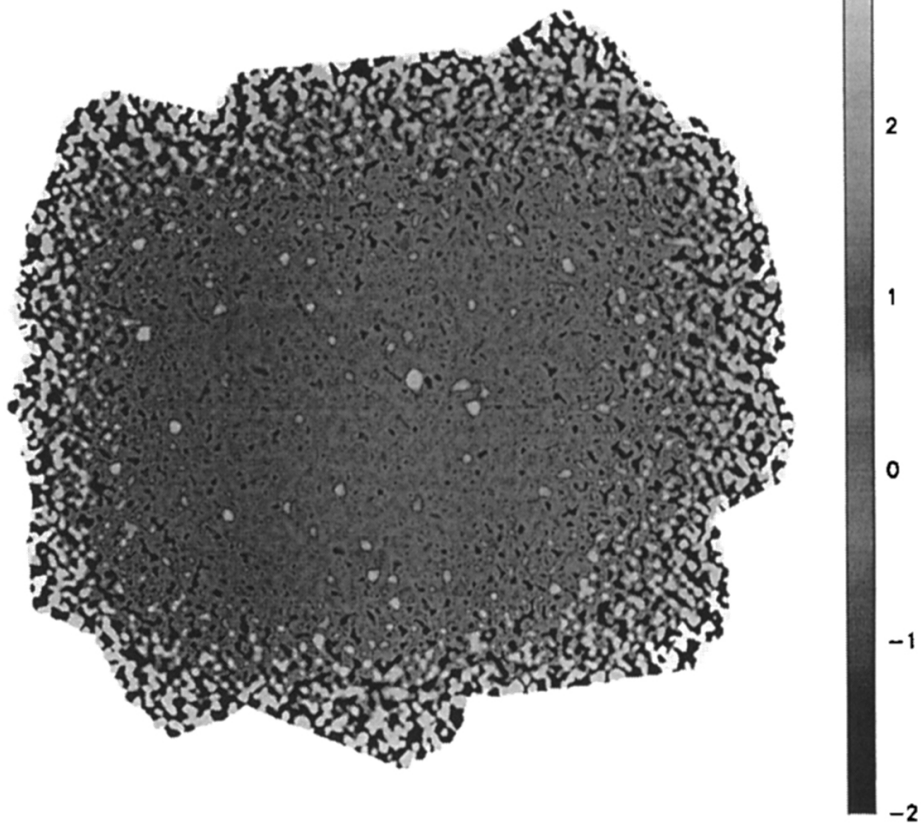

Figure $1 . \quad$ A preliminary $\approx 14$ arcmin diameter $1.2 \mathrm{~mm}$ MAMBO image of the NTT Deep Field. The rms noise is $0.5 \mathrm{mJy}$ in the image center, and rises toward the edges. At least 20 significant sources can be seen, including the strong $z=4.7$ quasar BR 1202-0725. During the last two winters we used MAMBO to obtain maps of three fields with a total area of about $300 \mathrm{arcmin}^{2}$ covered to a noise rms $<1 \mathrm{mJy}$. We found almost 100 point sources with fluxes above $1.5 \mathrm{mJy}$ (Bertoldi et al. 2000; Carilli et al. 2000; Menten \& Bertoldi 2000).

MAMBO source position. Since in our $7.5 \mu \mathrm{Jy}$ rms noise $20 \mathrm{~cm}$ VLA map on average we find only one $>5 \sigma$ source per $\operatorname{arcmin}^{2}$, chance alignments of radio and MAMBO sources are rare, and nearly all of the MAMBO-radio associations should be real. The radio identification of a MAMBO source determines its position within an arcsecond, and thereby allows a unique identification of any optical or near-IR counterpart, or as in most cases, the lack of one.

A radio identification also allows an approximate redshift determination. Relying on the tight correlation between the radio and the far-IR flux densities of star forming galaxies, Carilli \& Yun (1999) showed that the radio-to-(sub)mm flux ratio decreases with increasing redshift to such an extent that the observed value of this flux ratio can be used to estimate redshifts up to $z \approx 4$. Although contributions to the radio flux from an AGN, or systematically higher dust temperatures compared to local starburst galaxies would lead to an underestimate of the redshifts (Blain 1999), the radio-to-(sub)mm flux ratios of several dozen SCUBA and MAMBO sources provide a unique first look at their redshift distribution, placing most of them at $z \approx 1$ to 4 (Fig. 2). Including SCUBA/MAMBO sources for which upper limits to their radio fluxes are 
known, the redshift distribution shifts to slightly higher values, but it appears unlikely that there exists a dominant population of SCUBA/MAMBO sources at very high $(z>4)$ redshifts.

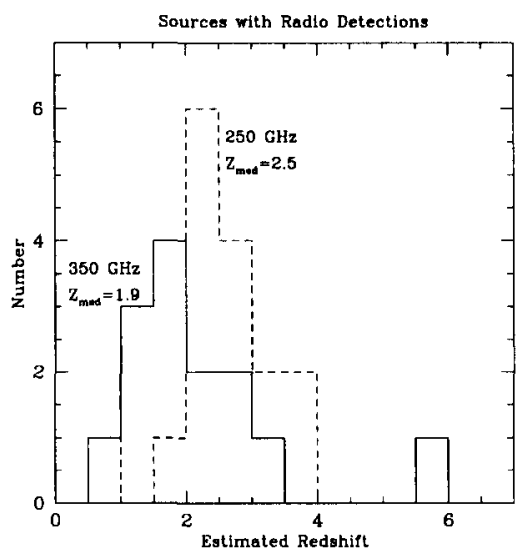

Figure 2. Redshift distribution for $250 \mathrm{GHz}(1.2 \mathrm{~mm})$ and $350 \mathrm{GHz}(850$ $\mu \mathrm{m})$ selected sources, based on the cmto-mm flux density ratio for sources with radio detections at $1.4 \mathrm{GHz}$, including redshift lower limits based on radio upper limits.

Figure 3 shows the preliminary cumulative source counts based on two of our three MAMBO deep fields, along with source counts determined from various SCUBA surveys. We relate the $250 \mathrm{GHz}$ flux densities to $350 \mathrm{GHz}$ flux densities using a scaling factor of 2.25 , applicable to a typical starburst galaxy at $z \approx 2.5$. We have included faint source counts in the regions within a $1^{\prime}$ radius of the cluster center assuming a mean gravitational magnification factor of 2.5 . The MAMBO and SCUBA counts agree well at intermediate flux densities, and they show a steepening of the distribution at $S_{350} \approx 10 \mathrm{mJy}$.

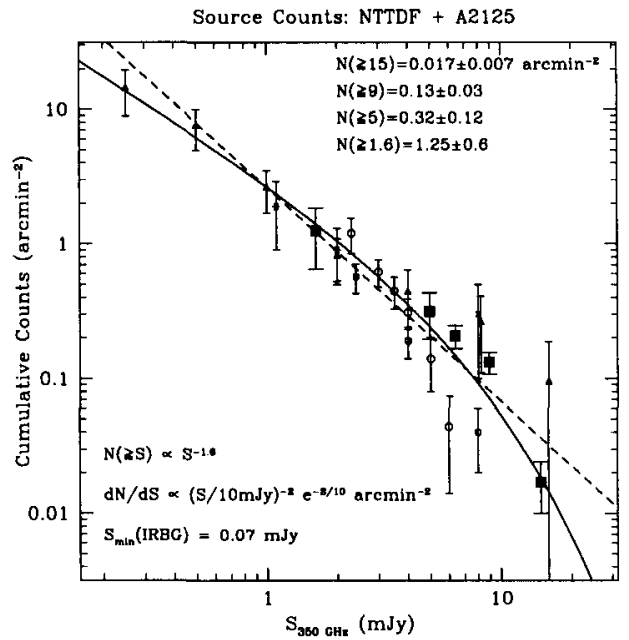

Figure 3. Preliminary cumulative source counts from two MAMBO fields as large solid squares, plus counts from various SCUBA surveys. The dashed curve is a power law of index -1.6. All of the data can be reasonably fit by an integrated Schechter-type luminosity function, with parameters as given on the plot.

What is the possible cause of a high brightness turnover of the (sub) mm background source counts? If these objects are indeed starbursts, then the implied star formation rates would be $>2000 M_{\odot} y r^{-1}$. Such extreme rates could only be sustained for some minimum (dynamical, free-fall, dissi- 
pation) timescale by the most massive galaxies. A turnover in the brightness distribution could thus indicate a turnover in the mass function of galaxies, or alternatively, it may be the signature of an upper limit to the luminosity of a starburst, an equivalent to the Eddington limit, determined by the energy output of the burst and the ability of the gas to dissipate energy rapidly enough to permit further mass infall or cloud collapse.

What is the nature of the (sub)mm background sources? It appears unlikely that a large fraction of them are dust-enshrouded QSOs, since Chandra observations failed to detect most of the targeted SCUBA sources (e.g. Fabian et al. 2000). However, two of the three SCUBA sources for which optical emission lines were seen show signatures of an active nucleus. The brightest source found in the MAMBO blind survey is an intermediate-redshift QSO, showing nonthermal emission at $\mathrm{mm}$ wavelengths. In a SCUBA mapping survey Knudsen et al. (2000) also find their brightest object to be a $z=2.8$ QSO. These two objects could be coincidental, but they do hint at a possible overlap of the (sub) mm background and the QSO populations.

Most likely, much of the energy emitted by the (sub)mm background sources arises from starbursts. Because then they produce a significant fraction of all stars in the Universe, and because they are more luminous than any starburst galaxy in the local universe, it is suggestive to think that they are elliptical galaxies seen at the time when they formed most of their stars. It would be of great interest to establish their exact redshift distribution. If they had formed over a wide range of redshifts, they probably formed through the hierarchical merging of smaller objects. If they formed in a narrow time interval at high redshift however, it would suggest that they formed through the collapse of single, massive primordial density enhancements. Our current estimate of their redshift distribution is consistent with the latter picture, the monolithic collapse of $10^{12} M_{\odot}$ structures, but this remains to be verified.

The MAMBO surveys are a collaborative effort also involving R. Zylka, L. Reichertz, A. Bertarini, D. Lutz, H. Dannerbauer, L. Tacconi, and R. Genzel.

\section{References}

Barger, A.J., Cowie, L.L., Sanders, D.B. 1999, ApJ 518, L5

Bertoldi, F., Carilli, C.L., Menten, K.M., et al. 2000, A\&A 360, 92

Blain, A.W. 1999, MNRAS 309, 955

Carilli, C.L., et al. 2000, in Deep Millimeter Surveys, astro-ph 0009298

Carilli, C.L., Yun, M.S. 1999, ApJ 513, L13

Eales, S.A., et al. 1999, ApJ 515, 518

Fabian, A.C. et al. 2000, MNRAS 315, 8

Hughes, D.H., et al. 1998, Nature 394, 241

Ivison, R., et al. 2000, MNRAS 315, 209

Knudsen, K.K., van der Werf, P.P., Jaffe, W. 2000, astro-ph 0009024

Lilly, S., et al. 1999, ApJ 518, 541

Menten, K.M., Bertoldi, F. 2000, Rev. Modern Astronomy, 13, 371

Smail, I., Ivison, R.J., Blain, A.W. 1997, ApJ 490, L5 